\title{
Mobility of D atoms on porous amorphous water ice surfaces under interstellar conditions
}

\author{
E. Matar, E. Congiu, F. Dulieu, A. Momeni ${ }^{\star}$, and J. L. Lemaire
}

\author{
Université de Cergy-Pontoise \& Observatoire de Paris, LERMA, UMR 8112 du CNRS, 95000 Cergy-Pontoise, France \\ e-mail: Elie.Matar@u-cergy.fr
}

Received 20 June 2008 / Accepted 23 October 2008

ABSTRACT

\begin{abstract}
Aims. The mobility of $\mathrm{H}$ atoms on the surface of interstellar dust grains at low temperature is still a matter of debate. In dense clouds, the hydrogenation of adsorbed species (i.e., CO), as well as the subsequent deuteration of the accreted molecules depend on the mobility of $\mathrm{H}$ atoms on water ice. Astrochemical models widely assume that $\mathrm{H}$ atoms are mobile on the surface of dust grains even if controversy still exists. We present here direct experimental evidence of the mobility of $\mathrm{H}$ atoms on porous water ice surfaces at $10 \mathrm{~K}$. Methods. In a UHV chamber, $\mathrm{O}_{2}$ is deposited on a porous amorphous water ice substrate. Then $\mathrm{D}$ atoms are deposited onto the surface held at $10 \mathrm{~K}$. Temperature-Programmed Desorption (TPD) is used and desorptions of $\mathrm{O}_{2}$ and $\mathrm{D}_{2}$ are simultaneously monitored.

Results. We find that the amount of $\mathrm{O}_{2}$ that desorbs during the TPD diminishes if we increase the deposition time of $\mathrm{D}$ atoms. $\mathrm{O}_{2}$ is thus destroyed by $\mathrm{D}$ atoms even though these molecules have previously diffused inside the pores of thick water ice. Our results can be easily interpreted if $\mathrm{D}$ is mobile at $10 \mathrm{~K}$ on the water ice surface. A simple rate equation model fits our experimental data and best fit curves were obtained for a D atom diffusion barrier of $22 \pm 2 \mathrm{meV}$. Therefore hydrogenation can take place efficiently on interstellar dust grains. These experimental results are consistent with most calculations and validate the hypothesis used in several models.
\end{abstract}

Key words. astrochemistry - ISM: atoms - dust, extinction - methods: laboratory

\section{Introduction}

Among the numerous molecules detected in different astrophysical environments, a large fraction is composed of hydrogenated species (Chang et al. 2007, and references therein). It has been established that dust grains play a major role in the hydrogenation of compounds (Herbst \& Klemperer 1973; Smith et al. 2006). In the dense interstellar medium where most of the hydrogenated species have been detected (especially during the star formation stage, Tegmark et al. 1997; Cazaux \& Tielens 2004; Cazaux \& Spaans 2004), grains are covered in a molecular mantle mainly composed of water ice. In all the theoretical chemical models that describe the hydrogenation of compounds on grains (Tielens \& Hagen 1982; Cuppen \& Herbst 2007), mobility of hydrogen is hypothesised, though some calculations on amorphous water surfaces (Smoluchowski 1981) and modelling of experimental data (Perets et al. 2005) rule out such a mobility. On the other hand, other calculations (Buch \& Zhang 1991; Masuda et al. 1998) and interpretations of experimental data (Hornekær et al. 2003) validate the hypothesis that $\mathrm{H}$ is mobile at $10 \mathrm{~K}$, a temperature supposed to be close to that of dust grains in interstellar dense clouds where hydrogenation takes place. We have already demonstrated that D atoms are mobile at $10 \mathrm{~K}$ (in our experiment time scale) on non-porous amorphous solid water (npASW) ices (Amiaud et al. 2007), but the porosity and the roughness of the water ice films grown at low temperatures $(10 \mathrm{~K})$ may considerably lower the D atom mobility.

Yet the debate upon the mobility of hydrogen atoms is not closed, and much controversy exists regarding the interpretation of experiments (Vidali et al. 2006; Dulieu 2006). If one

^ Present address: LCAM, Université Paris-Sud, Orsay, France. considers only the latest estimated value of $51 \mathrm{meV}$ (Perets et al. 2005 ) for the diffusion energy barrier of $\mathrm{H}$ on porous amorphous solid water (p-ASW) ice, the hopping time between two adjacent adsorption sites is about 10 million years at $10 \mathrm{~K}$. In such a context, no hydrogenation chemistry can occur on icy mantles of dust grains on a reasonable time scale. Hence, observational evidence of hydrogenated and deuterated species is a good argument for an experimental investigation of the mobility of $\mathrm{H}$ atoms.

The aim of this paper is to provide new experimental evidence that directly addresses - without the prism of a sophisticated model - the question of hydrogen mobility on p-ASW ice at $10 \mathrm{~K}$. By using $\mathrm{O}_{2}$ as a tracer of $\mathrm{D}$ mobility we present a set of experiments that are straightforward to interpret if $\mathrm{D}$ is mobile on the surface of porous amorphous water ice, as is expected from almost all calculations and included in several astrochemical models. In Sect. 2 we briefly describe the experimental set-up and procedures. In Sect. 3 we present our experimental results and explain them assuming that D is mobile on p-ASW ice at $10 \mathrm{~K}$. In Sect. 4, we describe a simple rate equation model that we used to fit our experimental results. In Sect. 5 we discuss other interpretations before concluding.

\section{Experimental section}

The FORmation of MOLecules in the InterStellar Medium (FORMOLISM) experimental set-up has been developed with the purpose of studying the reaction and interaction of atoms and molecules on surfaces simulating dust grains under interstellar conditions (the relevance of substrate, low density, and very low temperatures $\sim 10 \mathrm{~K}$ ). FORMOLISM is composed of an ultrahigh vacuum chamber with a base pressure of $\sim 10^{-10}$ mbar, a 
rotatable quadrupole mass spectrometer (QMS) and an oxygenfree high-conductivity copper sample holder. The sample holder is attached to the cold finger of a closed-cycle He cryostat and can be cooled to $8 \mathrm{~K}$. The temperature is measured with a calibrated silicon diode clamped on the sample holder and controlled by a Lakeshore 334 controller to $\pm 0.2 \mathrm{~K}$ with an accuracy of $\pm 1 \mathrm{~K}$ in the $8-400 \mathrm{~K}$ range. Reactants are introduced into the vacuum chamber via two separated triply differentially pumped beam lines aimed at the surface. The first one is used to deposit $\mathrm{O}_{2}$ while the second beam line is used to introduce the $\mathrm{D}$ atoms. This second beam line, in its first stage, consists of an air-cooled quartz tube surrounded by a microwave cavity for dissociating $\mathrm{D}_{2}$. To cool the $\mathrm{D}$ atoms exiting the dissociation region, the source is terminated with an aluminum nozzle connected to a closed cycle He cryostat that can be cooled to $\sim 25 \mathrm{~K}$. For the experiments presented here the $\mathrm{D}$ beam has a temperature of $50 \mathrm{~K}$ and a flux of $\sim 1 \times 10^{13} \mathrm{~cm}^{-2}$. The dissociation rate is constant at $\sim 50 \%$.

For this work we grow composite ice films of p-ASW on np-ASW. The sub-layer of np-ASW has a thickness of $\sim 50 \mathrm{ML}$ $\left(1 \mathrm{ML}=10^{15} \mathrm{~cm}^{-2}\right)$ and is grown at $120 \mathrm{~K}$ with a micro-channel array doser. The only purpose of the compact ice sub-layer is to isolate the ensuing p-ASW films from the $\mathrm{Cu}$ surface (Engquist et al. 1995). The p-ASW films are grown at $10 \mathrm{~K}$ on top of the np-ASW substrate. In the present study we use two different thickness of p-ASW, namely $20 \mathrm{ML}$ and $250 \mathrm{ML}$. The $20 \mathrm{ML}$ p-ASW film is grown from a background flux formed by controlling the $\mathrm{H}_{2} \mathrm{O}$ partial pressure in the vacuum chamber. Since ASW films deposited from ambient vapour take a long time to form and since a long time is needed to reach the base pressure again, the $250 \mathrm{ML}$ p-ASW films are deposited with the microchannel array doser. A previous calibration has shown that under these conditions we grow $0.33 \mathrm{ML}$ of ASW per second. Although p-ASW films grown by this method exhibit a lower degree of porosity, the greater thickness ensures a more complex pore structure than that of thin p-ASW ices grown by background dosing. After the growth of the composite ice films, they are annealed to $70 \mathrm{~K}$ to avoid any subsequent morphological changes. Although the porous structure is not completely destroyed by the annealing process, this reduces irreversibly the porosity of the p-ASW films (Kimmel et al. 2001a,b). This is verified by looking at the shift in temperature of the $\mathrm{D}_{2}$ desorption peak as described in Hornekær et al. (2005).

Our experimental procedures are the following:

- After the preparation of a stable and pure $\mathrm{O}_{2}$ beam, the p-ASW surface held at $10 \mathrm{~K}$ (or $25 \mathrm{~K}$ in another set of experiments) is exposed to $0.5 \mathrm{ML}$ of $\mathrm{O}_{2}$ molecules. The flux was previously calibrated using TPD by determining the $\mathrm{O}_{2}$ exposure time required to saturate the $\mathrm{O}_{2}$ monolayer on np-ASW ice (Kimmel et al. 2001b).

- The p-ASW substrate kept at $10 \mathrm{~K}$ is then exposed to varying amounts of cold $\mathrm{D}$ atoms $(50 \mathrm{~K})$ ranging from 0 exposed monolayers (EML) to 2 EML. We use EML and not directly ML because in the case of light molecules the sticking coefficient is not necessarily unity. Thus fewer molecules than those actually impinging on the surface are adsorbed. Moreover, in the case of p-ASW ice more than $1 \mathrm{ML}$ of $\mathrm{D}_{2}$ can be adsorbed on the surface, and still the coverage can be low, since the effective surface area is very large due to the 3D conformation of the substrate (Amiaud et al. 2006).

- Finally, the QMS is placed in front of the surface and TPD mass spectra of $\mathrm{D}_{2}$ and $\mathrm{O}_{2}$ are recorded simultaneously. All TPDs are done with a linear heating rate of

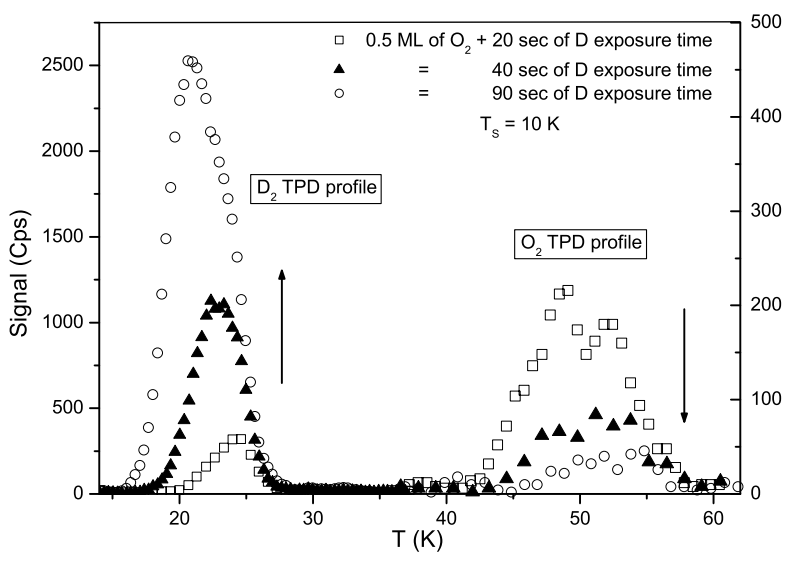

Fig. 1. TPD profiles of $\mathrm{D}_{2}$ and $\mathrm{O}_{2}$ after deposition of $0.5 \mathrm{ML}$ of $\mathrm{O}_{2}$ and followed by different deposition times of $\mathrm{D}$ at $T_{S}=10 \mathrm{~K}$. The arrows emphasise that the quantity of desorbed $\mathrm{O}_{2}$ decreases as the amount of exposed D grows.

$10 \mathrm{~K} / \mathrm{min}$ starting from $10 \mathrm{~K}$ up to a temperature above which the $\mathrm{O}_{2}$ molecules have completely desorbed $(\sim 65 \mathrm{~K})$ (see Fig. 1).

- The same series of experiments are also done by depositing $\mathrm{D}$ and $\mathrm{D}_{2}$ followed by $\mathrm{O}_{2}$.

\section{Results}

We find that $\mathrm{O}_{2}$ is only sensitive to $\mathrm{D}$ and not to $\mathrm{D}_{2}$. When $\mathrm{D}$ is deposited on the surface and then exposed to $\mathrm{O}_{2}$, we find that the amount of desorbed $\mathrm{O}_{2}$ is constant (within the experimental error bars $(<10 \%)$ ) irrespective of the $\mathrm{D}$ amounts. This series of experiments was done in order to rule out the possibility that $\mathrm{D}$ atoms were mobile only during the heating phase of the TPD. Indeed these results demonstrate that D atoms that adsorb on the ice surface diffuse immediately and either react with each other or desorb before $\mathrm{O}_{2}$ is deposited. The amounts of desorbing $\mathrm{O}_{2}$ are the same regardless of the amounts of D atoms deposited on the surface. Hence, in what follows, we restrict ourselves to experiments where $\mathrm{O}_{2}$ is deposited first and then followed by various $\mathrm{D}$ exposure times.

Figure 1 shows the TPD profiles of $\mathrm{O}_{2}$ and $\mathrm{D}_{2}$ after the exposure of $0.5 \mathrm{ML}$ of $\mathrm{O}_{2}$ to 20,40 and $90 \mathrm{~s}$ of $\mathrm{D}$ irradiation. The $\mathrm{O}_{2}$ peak area decreases with the $\mathrm{D}$ deposition time and this proves that D atoms "consume" $\mathrm{O}_{2}$ molecules adsorbed on the surface by reacting with them. Figure 2 displays the normalised TPD peak areas of $\mathrm{O}_{2}$ and $\mathrm{D}_{2}$, as a function of $\mathrm{D}$ exposure time (lower axis) or D EML (upper axis). Figures 1 and 2 show clearly that the quantity of desorbing $\mathrm{O}_{2}$ decreases with the increase of the exposed dose of D. A sudden change in the slope of the monotonic decrease occurs after $\sim 0.5$ EML of D exposure. At first, the drop of $\mathrm{O}_{2}$ that desorbs from the surface occurs rapidly, then it becomes much slower. On the other hand, as expected, the amount of $\mathrm{D}_{2}$ desorbing from the p-ASW surface increases with the exposure time of D (Fig. 2). Yet, in this case too, we observe that a change in the slope of the $\mathrm{D}_{2}$ desorption raise takes place almost exactly after $\sim 0.5$ EML of D exposure. Here the increase of $\mathrm{D}_{2}$ TPD peak areas is slightly accelerated after the slope change.

These observations can be explained as follows: as D atoms are deposited onto the p-ASW sample, two competing mechanisms occur, namely $\mathrm{O}_{2}$ destruction and $\mathrm{D}_{2}$ formation. If $\mathrm{D}$ atoms are mobile, they are likely to encounter either another 


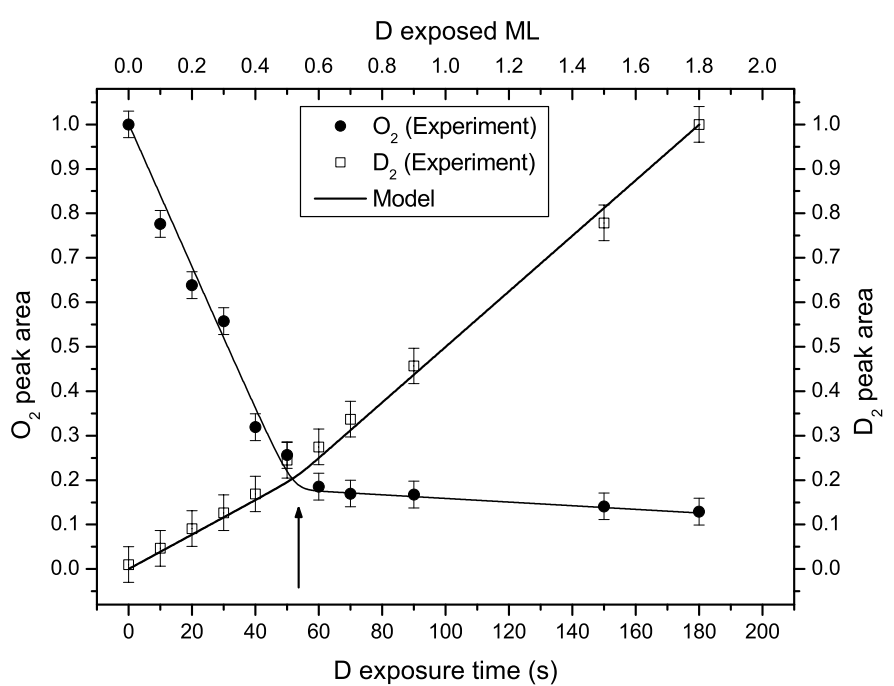

Fig. 2. Normalised $\mathrm{O}_{2}$ (circles) and $\mathrm{D}_{2}$ (squares) TPD peak areas following different $\mathrm{D}$ exposures on $20 \mathrm{ML}$ of p-ASW ice. $\mathrm{O}_{2}$ normalisation was performed with respect to the TPD peak area corresponding to $0.5 \mathrm{ML}$ of $\mathrm{O}_{2}$ alone and that of $\mathrm{D}_{2}$ was made with respect to the TPD peak area corresponding to 1.8 EML of D. $\mathrm{O}_{2}$ and D both deposited at a surface temperature of $10 \mathrm{~K}$. The solid lines represent the model results (see text).

$\mathrm{D}$ atom to form $\mathrm{D}_{2}\left(\mathrm{D}+\mathrm{D}\right.$ formation) or an $\mathrm{O}_{2}$ molecule and react with it $\left(\mathrm{O}_{2}\right.$ destruction $)$. It is significant that in both cases of $\mathrm{O}_{2}$ and $\mathrm{D}_{2}$ desorptions, the change in slope occurs after the same amount of $\mathrm{D}$ exposure time (see arrow in Fig. 2). This suggests that after $\sim 50 \mathrm{~s}(0.5 \mathrm{EML})$ of $\mathrm{D}$ irradiation, when most $\mathrm{O}_{2}$ has been destroyed, a greater number of $\mathrm{D}$ atoms are available to form $\mathrm{D}_{2}$. The presence of a rapid regime of $\mathrm{O}_{2}$ destruction ( $80 \%$ of the initial dose destroyed by 0.5 EML of D) and of a slow regime (for the remaining $20 \%$ of the $\mathrm{O}_{2}$ initial dose) can be ascribed to the non-perfect overlapping of the two beams. It has been checked that the two beams have in fact $\sim 80 \%$ overlap on the surface.

We then performed the same experiment using a thicker p-ASW ice film in order to check whether the destruction of $\mathrm{O}_{2}$ on a $20 \mathrm{ML}$ p-ASW ice substrate is actually due to mobile $\mathrm{D}$ atoms being able to scan the porous surface. This time, on $250 \mathrm{ML}$ p-ASW films, we deposited $\mathrm{O}_{2}$ at a higher temperature $(25 \mathrm{~K})$, to favour $\mathrm{O}_{2}$ mobility and to have $\mathrm{O}_{2}$ not only adsorbed on the surface of the film but also deeper into the porous structure. The results are shown in Fig. 3. Plain circles represent the yield of desorbed $\mathrm{O}_{2}$ after cooling the surface to $10 \mathrm{~K}$ and then irradiating with $0,50,100$ and $150 \mathrm{~s}$ of D exposure at $10 \mathrm{~K}$, while empty circles show the case of desorption of $\mathrm{O}_{2}$ initially deposited at $10 \mathrm{~K}$ and then exposed to the same D exposure times. First, we notice in these experiments that the $\mathrm{O}_{2}$ destruction rate is much slower than in the case of a $20 \mathrm{ML}$ p-ASW substrate. We then only observe the first part of the curve in Fig. 2 at a slower rate. This can be due to the fact that the roughness is greater in the case of a $250 \mathrm{ML}$ substrate. The structures (holes, pores, piles ...) are more pronounced. The total accessible surface is also higher and subsequently the average density of $\mathrm{O}_{2}$ is reduced. In this case $\mathrm{D}_{2}$ formation is more favoured. Secondly, we find that if $\mathrm{O}_{2}$ is deposited at $25 \mathrm{~K}$, the destruction rate is even lower. This can be due to the fact that at $25 \mathrm{~K}$ $\mathrm{O}_{2}$ is more mobile on the surface of the ASW ice than at $10 \mathrm{~K}$. Therefore $\mathrm{O}_{2}$ molecules are spread over all the accessible porous surface. $\mathrm{O}_{2}$ density is again lower and $\mathrm{D}_{2}$ formation is favoured.

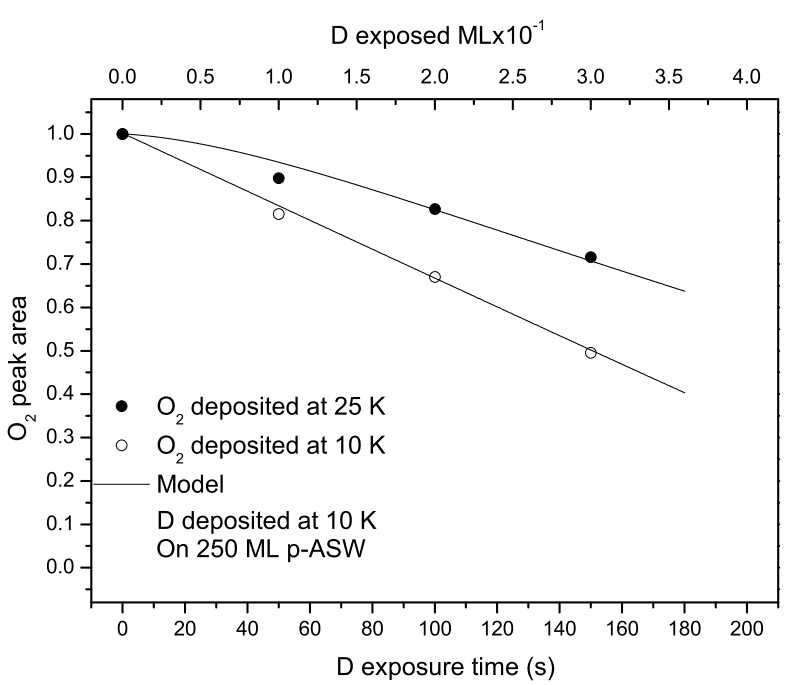

Fig. 3. Comparison between normalised TPD peak areas of 0.5 ML of $\mathrm{O}_{2}$ deposited at $10 \mathrm{~K}$ (blank dots) and $25 \mathrm{~K}$ (black dots) and then irradiated with $0,50,100$ and $150 \mathrm{~s}$ of D atoms on $250 \mathrm{ML}$ of p-ASW ice. The solid line represents the model results (see text).

We also notice the disappearance of the elbow that was present in Fig. 2 at 0.5 EML of D. It is due to the fact that we should have exposed the surface to longer times of $\mathrm{D}$ atoms in order to reach $80 \%$ destruction of the adsorbed $\mathrm{O}_{2}$ to observe it. Finally we have checked that all the $\mathrm{O}_{2}$ deposited at $25 \mathrm{~K}$ disappears, after very large exposure times $(600 \mathrm{~s})$, even on the $250 \mathrm{ML}$ p-ASW film.

\section{Model and data fit}

A very simple model based upon the formalism of Katz et al. (1999) was used to fit the experimental results already obtained and shown in Fig. 2. If $F_{\text {tot }}$ is the initial beam flux of $\mathrm{D}_{2}$, after the dissociation $F_{\text {tot }}$ is divided into a flux of $\mathrm{D}, F_{\mathrm{D}}$, and a flux of $\mathrm{D}_{2}$, $F_{\mathrm{D} 2}$. Both fluences on the surface are determined knowing the sticking probabilities of $\mathrm{D}_{2}$ and of $\mathrm{D}, S_{\mathrm{D} 2}$ and $S_{\mathrm{D}}$ respectively, and the dissociation rate $\tau$ at a beam temperature of $50 \mathrm{~K}$. The fluxes are given by:

$F_{\mathrm{D}}=2 \tau P_{\mathrm{D}} F_{\text {tot }}$ and; $F_{\mathrm{D}_{2}}=(1-\tau) P_{\mathrm{D}_{2}} F_{\text {tot }}$.

Taking into account the porosity of the ice surface, the surface density of $\mathrm{D}, \mathrm{D}_{2}$ and $\mathrm{O}_{2}$, and depending on the diffusion barrier $E_{\text {diff }}$ of $\mathrm{D}$ atoms on the surface, this model simulates the number of $\mathrm{D}+\mathrm{D}$ reactions as well as the number of $\mathrm{D}+\mathrm{O}_{2}$ reactions. As Figs. 2 and 3 clearly show, our simple model is able to reproduce the experimental results under the same conditions, namely, dissociation efficiency of the $\mathrm{D}_{2}$ beam, sticking probabilities of $\mathrm{D}$ and $\mathrm{D}_{2}$ (measured at a given beam temperature, Matar et al., in preparation), and porosity of the ice. A best fit of the experimental data has given a diffusion barrier of $22 \pm 2 \mathrm{meV}$.

\section{Discussion}

In the previous section we proposed a direct interpretation of our experimental data assuming that D is mobile at $10 \mathrm{~K}$. But other hypotheses also should be discussed in the light of these new experimental data, in particular the possible thermally induced mobility of the atoms. This process implies that atoms are not mobile at $10 \mathrm{~K}$ and that they require higher temperature to 
move and react. If atoms are not mobile at $10 \mathrm{~K}$, they should stay in the vicinity of the external surface, especially under our experimental conditions where $\mathrm{D}$ atoms have a kinetic temperature of $50 \mathrm{~K}$ (in the beam) which considerably reduces the mobility of $\mathrm{D}$ atoms resulting from the hot atom (Harris-Kasemo) mechanism.

The total number of $\mathrm{D}$ atoms sent to the surface can be quite high $\left(2 \times 10^{15} \mathrm{~cm}^{-2}\right.$, i.e. $\left.2 \mathrm{ML}\right)$ which corresponds to a value of full coverage of the external surface. Consequently, if atoms are not mobile, the surface density becomes high, and two further mechanisms invoked in the literature should become nonnegligible. One is the direct Eley-Ridel formation mechanism for $\mathrm{D}_{2}$ (an adsorbed atom forms a molecule with an atom incoming from the gas phase, a fraction of the molecules formed is then directly released in the gas phase), the second is the rejection mechanism (Perets et al. 2005) (adsorbed atoms prohibit atoms from the gas phase sticking on the surface). In both cases a fraction of $\mathrm{D}$ or $\mathrm{D}_{2}$ evaporates during the exposure and therefore does not desorb later during the heating ramp. If so, $\mathrm{D}_{2}$ should not be proportional to the exposure, which is not the case in Fig. 2 where we observe proportionality. We can thus conclude that either the surface density does not increase to reach a value of the coverage close to unity or the two cited mechanisms (the Eley-Ridel and rejection mechanisms) are not efficient. However, it is unlikely that the E-R mechanism does not occur, therefore the coverage remaining low implies that $\mathrm{D}$ diffuses through the pores.

Calculations (Buch \& Czerminski 1991) show that the adsorption energy of $\mathrm{H}$ on a water cluster and the height of the energy barrier between two adjacent sites are both described by large distributions, the latter being peaked in the $19.4-34.5 \mathrm{meV}$ range. This means that the mobility is site dependent. Some deep adsorption sites can bind atoms more than others and release them less easily in the gas phase. Thus it is possible that a fraction of the atoms are not mobile at $10 \mathrm{~K}$ on the time scale of the experiment, and require thermal activation to react. Our experiments show that globally D atoms are able to penetrate the porous structure of the ice. In fact, we find a non-infinite $\mathrm{D}$ atom mobility $\left(E_{\mathrm{diff}}=22 \pm 2 \mathrm{meV}\right.$, in agreement with calculations). This value is about half the one obtained by Perets et al. (2005). The discrepancy between the two results stems probably from different assumptions made on the surface coverage. Despite the fact that the atom fluence is about the same in both works (up to $2 \mathrm{ML}$ of $\mathrm{D}$ ), Perets et al. assume that their coverage is always less than $1 \%$. Our results do not show if this mobility is dominated by thermal hopping or tunnelling. More experiments should be done at lower surface temperature (below $8 \mathrm{~K}$ ) to fully understand how this mobility occurs. Another aspect of these experiments concerns the efficiency of the $\mathrm{O}_{2}+\mathrm{D}$ reaction in comparison with the $\mathrm{D}+\mathrm{D}$ reaction. We can see that $\mathrm{O}_{2}+\mathrm{D} \rightarrow$ $\mathrm{O}_{2} \mathrm{D}$ is a very efficient reaction (Miyauchi et al. 2008; Ioppolo et al. 2008), because the proportion of $\mathrm{D}$ required to destroy $\mathrm{O}_{2}$ is close to 1 , at the accuracy of our flux estimation $(\sim 50 \%)$, because $\mathrm{O}_{2}+\mathrm{D}$ is believed to have a low energy barrier (Walch et al. 1988). In our experiments the fact that the $\mathrm{O}_{2}+\mathrm{D}$ reaction seems to be favoured in comparison with the $\mathrm{D}+\mathrm{D}$ reaction when the exposition is low does not signify that the $\mathrm{D}+\mathrm{D}$ reaction is less efficient because of an activation barrier. Indeed, our model assumes that both $\mathrm{D}+\mathrm{D}$ and $\mathrm{O}_{2}+\mathrm{D}$ reactions have $100 \%$ efficiency. Once again, if atoms are mobile, the density of $\mathrm{D}$ atoms stays low because they are consumed by the $\mathrm{O}_{2}$ population. It is only when the density of $\mathrm{D}$ atoms is equal to that of $\mathrm{O}_{2}$ atoms that the production of $\mathrm{D}_{2}$ can be effective. This consideration allows us to conclude that at any moment in our experiment, the density of
$\mathrm{D}$ atoms is low, initially because they are consumed by $\mathrm{O}_{2}$ and subsequently because they form $\mathrm{D}_{2}$.

At the end of the experiment, when the ice is sublimated we detect a high fraction of $\mathrm{HDO}$ and not $\mathrm{D}_{2} \mathrm{O}$ owing to thermally activated $\mathrm{H} / \mathrm{D}$ exchanges (Smith et al. 1997). Therefore $\mathrm{O}_{2}$ is one of the possible precursors for water formation. Finally these experiments show that no $\mathrm{O}_{2}$ should survive on grains in dark interstellar clouds. Apart from specific detection limitations, it can be a fundamental reason for the non detection of $\mathrm{O}_{2}$ on icy grains in dark clouds.

\section{Conclusions}

We have reported the results of experiments on the mobility of deuterium at $10 \mathrm{~K}$ on porous ASW ice surfaces under interstellar conditions, using a temperature programmed desorption technique. Beams of $\mathrm{O}_{2}$ and $\mathrm{D}$ were irradiated on the surface of an p-ASW ice film and the mobility of D atoms at $10 \mathrm{~K}$ was investigated via their property of reacting with the $\mathrm{O}_{2}$ molecules. By using a simple rate equation model we found that the $\mathrm{D}$ atoms diffusion barrier at $10 \mathrm{~K}$ is $22 \pm 2 \mathrm{meV}$. Our experimental results demonstrate that D atoms are mobile on ASW ice surfaces at $10 \mathrm{~K}$, and validate the chemical models that hypothesized the mobility of $\mathrm{H}$ atoms at $10 \mathrm{~K}$.

Acknowledgements. The authors would like to thank L. E. Kristensen, V. Pirronello and O. Biham for fruitful discussions. We acknowledge the support of the national PCMI program founded by the CNRS, as well as the strong financial support from the Conseil Régional d'Ile de France through SESAME programs (E1315 and I-07-597R), the Conseil Général du Val d'Oise and the Agence Nationale de la Recherche.

\section{References}

Amiaud, L., Fillion, J. H., Baouche, S., et al. 2006, J. Chem. Phys., 124, 094702 Amiaud, L., Dulieu, F., Fillion, J.-H., Momeni, A., \& Lemaire, J. L. 2007, J. Chem. Phys., 127, 4709

Buch, V., \& Czerminski, R. 1991, J. Chem. Phys., 95, 6026

Buch, V., \& Zhang, Q. 1991, ApJ, 379, 647

Cazaux, S., \& Spaans, M. 2004, ApJ, 611, 40

Cazaux, S., \& Tielens, A. G. G. M. 2004, ApJ, 604, 222

Chang, Q., Cuppen, H. M., \& Herbst, E. 2007, A\&A, 469, 973

Cuppen, H. M., \& Herbst, E. 2007, ApJ, 668, 294

Dulieu, F. 2006, Farady Discuss., 133, 191

Engquist, I., Lundstroem, I., \& Liedberg, B. 1995, J. Phys. Chem., 99, 12257

Herbst, E., \& Klemperer, W. 1973, ApJ, 185, 505

Hornekær, L., Baurichter, A., Petrunin, V. V., Field, D., \& Luntz, A. C. 2003 , Science, 302, 1943

Hornekær, L., Baurichter, A., Petrunin, V. V., et al. 2005, J. Chem. Phys., 122, 4701

Ioppolo, S., Cuppen, H. M., Romanzin, C., van Dishoeck, E. F., \& Linnartz, H. 2008, ApJ, 686, 1474

Katz, N., Furman, I., Biham, O., Pirronello, V., \& Vidali, G. 1999, ApJ, 522, 305 Kimmel, G. A., Dohnálek, Z., Stevenson, K. P., Smith, R. S., \& Kay, B. D. 2001a, J. Chem. Phys., 114, 5295

Kimmel, G. A., Stevenson, K. P., Dohnálek, Z., Smith, R. S., \& Kay, B. D. 2001b, J. Chem. Phys., 114, 5284

Masuda, K., Takahashi, J., \& Mukai, T. 1998, A\&A, 330, 773

Miyauchi, N., Hidaka, H., Chigai, T., et al. 2008, Chem. Phys. Lett., 456, 27

Perets, H. B., Biham, O., Manicó, G., et al. 2005, ApJ, 627, 850

Smith, I. W. M., Sage, A. M., Donahue, N. M., Herbst, E., \& Quan, D. 2006, Faraday Discuss., 133, 137

Smith, R., Huang, C., \& Kay, B. 1997, J. Phys. Chem. B, 101, 6123

Smoluchowski, R. 1981, Ap\&SS, 75, 353

Tegmark, M., Silk, J., Rees, M. J., et al. 1997, ApJ, 474, 1

Tielens, A. G. G. M., \& Hagen, W. 1982, A\&A, 114, 245

Vidali, G., Roser, J. E., Ling, L., et al. 2006, Faraday Discussions, 133, 125

Walch, S. P., McMichael Rohlfing, C., Melius, C. F., \& Bauschlicher, Jr., C. W. 1988, J. Chem. Phys., 88, 6273 